AIAA 99-0046

\title{
Non-Destructive Evaluation of Wind Turbine Blades Using an Infrared Camera ${ }^{*} \dagger$
}

\author{
Alan G. Beattie $\S$ \\ Mark Rumsey \\ Sandia National Laboratories \\ PO Box 5800 \\ Albuquerque, NM 87185
}

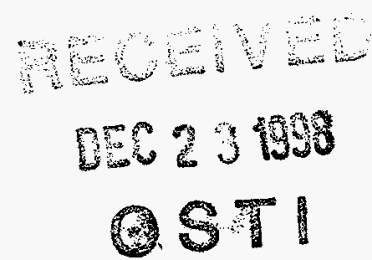

\begin{abstract}
The use of a digital infrared thermography camera as a non-destructive evaluation (NDE) tool was explored in two separate wind turbine blade fatigue tests. The first test was a fatigue test of part of a 13.1 meter wood-epoxy-composite blade. The second test was on a 4.25 meter pultruded fiber glass blade section driven at several mechanical resonant frequencies. The digital infrared camera can produce images of either the static temperature distribution on the surface of the specimen, or the dynamic temperature distribution that is in phase with a specific frequency on a vibrating specimen. The dynamic temperature distribution (due to thermoelastic effects) gives a measure of the sum of the principal stresses at each point on the surface. In the woodepoxy-composite blade fatigue test, the point of ultimate failure was detected long before failure occurred. The mode shapes obtained with the digital infrared camera, from the resonant blade tests, were in very good agreement with the finite-element calculations. In addition, the static temperature images of the resonating blade showed two areas that contained cracks. Close-up dynamic infrared images of these areas showed the crack structure that agreed with subsequent dye-penetrant analysis.
\end{abstract}

\section{Introduction}

The National Renewable Energy Laboratory (NREL) has been conducting a series of static and fatigue load tests on full-scale wind turbine blades at the National Wind Technology Center (NWTC), located south of Boulder, Colorado.
The object of these tests is to aid the wind energy industry and the national labs in understanding the static and fatigue characteristics of wind turbine blades. To date the prime methods of instrumentation used in these tests are strain gauges and, occasionally, acoustic emission NDE monitoring. These methods can require a large number of sensors and a maze of wiring. A noncontact wide-area method of analysis that is reliable and simple to use would be of great value in these test programs.

Thermoelastic stress analysis ${ }^{1}$, using an infrared thermography camera, would appear to be a viable candidate for a non-contact wide-area technique. Thermoelastic stress occurs when oscillating surface stress variations give rise to small oscillating surface temperature gradients. Up to a few years ago, the only frequency dependent thermal camera was the SPATE ${ }^{2}$ system where the surface is scanned by a rotating mirror and the image detected by a single linear infrared detector array. This system is adequate for small parts, but has some disadvantages when used on large objects. The development of the digital infrared camera system ${ }^{3}$, with a $128 \times 128$ detector array, with the appropriate lens combination, presents a more flexible thermoelastic measuring system for use with large objects.

The purpose of this study was to determine the value of a digital infrared camera in the testing of fullscale wind turbine blades.

A digital infrared camera was used in two separate fatigue tests. The first test was a fatigue test of part of a

* Sandia is a multi-program laboratory operated by Sandia Corporation, a Lockheed Martin Company, for the United States Department of Energy under Contract DE-ACO4-94AL85000.

$\dagger$ This paper is declared a work of the U.S. Government and is not subject to copyright protection in the United States.

$\$$ The mention of trade or manufacture names, in this paper, is made for information only and does not imply an endorsement, recommendation, or exclusion by the Sandia National Laboratories.

$\S$ Alan G. Beattie is now with Physical Acoustics Corp., Box 462, Corrales, NM 87048. 


\section{DISCLAIMER}

This report was prepared as an account of work sponsored by an agency of the United States Government. Neither the United States Government nor any agency thereof, nor any of their employees, make any warranty, express or implied, or assumes any legal liability or responsibility for the accuracy, completeness, or usefulness of any information, apparatus, product, or process disclosed, or represents that its use would not infringe privately owned rights. Reference herein to any specific commercial product, process, or service by trade name, trademark, manufacturer, or otherwise does not necessarily constitute or imply its endorsement, recommendation, or favoring by the United States Government or any agency thereof. The views and opinions of authors expressed herein do not necessarily state or reflect those of the United States Government or any agency thereof. 


\section{DISCLAIMER}

\section{Portions of this document may be illegible in electronic image products. Images are produced from the best available original document.}


13.1 meter AWT-26/27 (Advanced Wind Turbine, Inc.) wood-epoxy-composite wind turbine blade. The test was conducted at the NREL/NWTC test facility. The second fatigue test was of a 4.25 meter long section of a pultruded fiber reinforced plastic wind turbine blade, mechanically driven at several of the blade's resonate frequencies. This test was conducted in a modal test lab at the Sandia National Laboratories in Albuquerque, New Mexico. The modal patterns of the blade, detected by the digital infrared camera, were compared to finiteelement calculations.

\section{Instrumentation}

The Stress Photonics Inc. DeltaTherm $1000^{\mathrm{TM}}$ is a modern digital infrared camera specifically designed for acquiring thermoelastic data. The camera is capable of taking steady-state (DC) surface temperature measurements. It can also make single frequency $(\mathrm{AC})$ surface temperature measurements at frequencies in the range of $1 / 4$ to $4000 \mathrm{Hertz}$. The camera records an image that is the output of an array (128 pixels by 128 pixels) of IR detectors. Each pixel in the array is the output from one detector. The scan rate is 430 images per second. For steady-state measurements, each pixel is simply averaged over a specified time. For single frequency measurements, the pixels in each image are multiplied by functions electronically derived from a reference signal to give in-phase and out-of-phase images. The phase relative to the reference signal can be set either automatically for maximum separation between the two images or arbitrarily by the operator. After the multiplications, the in-phase and out-of-phase signals are time averaged. For reference frequencies greater than the nyquest frequency $(215 \mathrm{~Hz})$, an anti-aliasing signal processing method is used. The study of wind turbine blades does not require the high frequency capabilities of the camera.

Steady-state (DC) differential measurements were made by subtracting from the sample image an unfocused image of a black aluminum reference block that is close to the operating temperature of the sample. This procedure removes variations in the detector sensitivities and allows temperature resolutions of approximately $0.01^{\circ} \mathrm{F}$. The single frequency measurements are made by summing the modified values from each detector (pixel) over time and subtracting out the average steady-state value of all the pixels. This procedure is the equivalent of using a lock-in amplifier. Sufficiently long averaging times ( $\sim 2$ minutes) will allow a few milli-degrees $\mathrm{F}$ oscillating temperature component resolution.

The DeltaTherm $1000^{\mathrm{TM}}$ camera is typically mounted on a tripod, and connected to the signal proc- essing and analysis electronics with a long umbilical cord. In operation, liquid nitrogen, contained in a builtin reservoir, is used to cool the detector array in the camera. For the best results the camera's optical axis should be normal to the moving surface being monitored, but the open top-loading liquid nitrogen reservoir restricts the tile angle. Practically, the camera is restricted to an angle of about $\pm 60^{\circ}$ to the horizontal. The pultruded blade was driven in a horizontal direction and the tripod-mounted camera was used without a problem. However, for the AWT blade fatigue test, the blade was driven in a vertical direction. For this test, the camera was mounted horizontally on the floor, under the blade, and the infrared radiation was reflected into the camera by means of a front-surface mirror that was held at $45^{\circ}$ angle to the floor. This setup is shown in figure 1 .

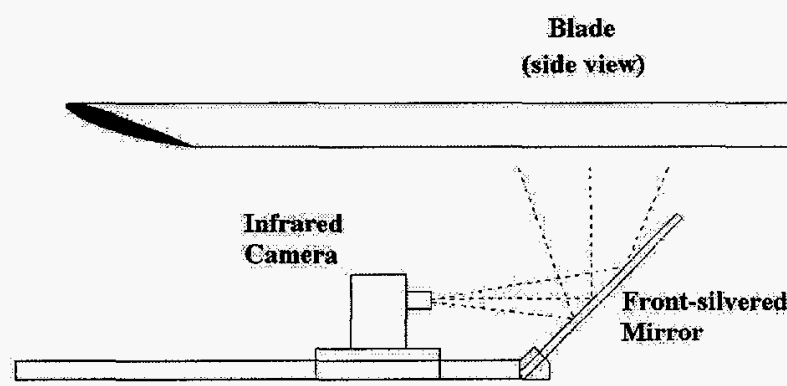

Figure 1. Diagram of the floor-mounted fixture used by the infrared camera.

A primary concern in using an infrared camera with wind turbine blades was the emissivity of the blade surface. The prospect of painting a blade several meters long and a few meters wide was daunting. Both blades had epoxy surfaces. The visible color of both was approximately tan. Initial experiments were conducted to see the difference between the camera response to the natural epoxy surface and a small section of the blade surface spray painted flat-black. For motion parallel to the optical axis (towards and away from the camera), differences in response were almost non-existent. The differences in the surface emissivities at the infrared frequencies detected (3-5 micrometers) were, at worst, a few percent.

A problem that did appear was that the shiny white gelcoat epoxy surface reflected infrared radiation while the flat-black painted surface did not. This did not effect the pultruded blade test where the amplitude of the surface motion was small and parallel to the optical axis. However, in the wood-epoxy-composite blade fatigue test the blade motion had amplitudes of up to $\pm 25 \mathrm{~cm}$, with surface motion variations normal to the optical-axis of a few millimeters. (The amount of motion varies along the blade. The blade root is mounted to a fixed seismic mass -- there is little to no motion at 
this point; the blade tip will have the largest motion variations.) This motion caused continuous changes in the angle between the mirror and the blade surface. For the steady-state (DC) thermal measurements, this only produced a slight smearing of the images. In the stress (AC) measurements, the combination of the lock-in detection, surface motion, and curvature of the blade along one axis, produced distortions or mottling in the image due to reflected infrared radiation off the shiny blade surface. Almost all infrared sources in the vicinity of the blade produced reflections, but the real problems arose from those with sharply defined edges. Black felt cloth on the floor, below the camera, greatly reduced the effect of reflections in the images.

An important note concerning the images in this paper. The output thermal images of the digital infrared camera are rich in colors. This document can only reproduce the thermal images in shades of gray. Therefore, some information and a significant amount of visual impact have been lost in the figures.

\section{$\underline{\text { AWT Blade Test }}$}

The 13.1 meter AWT blade is constructed of woodepoxy laminates with a root attachment structure consisting of 15 female-threaded inserts bonded into the wood-epoxy root section of the blade. Several previous blades of this design had been tested at the NREL/NWTC. All had failed near the root-insert interfaces. The blade itself was sealed with epoxy on the inner and outer surfaces and the outer layer was a white gelcoat. Because of the length of the blade, there were several staggered scarf joints in the wood laminates. The root of the blade was attached to a steel hub extender that in turn was mounted to a large steel seismicmass base structure. The blade was mounted in a horizontal position with the chord plane also horizontal (flapwise test). The hydraulic actuator was mounted on the floor and attached at the cut-off end of the blade. The last 5.1 meters of the blade were removed in order to mount a single point actuator at its end. The load was applied at $2.0 \mathrm{Hertz}$, and was always positive ranging from $647 \mathrm{lbs}$. to $6467 \mathrm{lbs}$.

Figure 2 is a steady-state (DC) thermal image of an area at the blade root taken after cyclic loading for over 12 hours. Roughly 100,000 continuous cycles had been applied to the blade at this point. The metal label on the blade shows at the top of the blade and the small black square at the bottom is a $1 / 2$ inch one-foot fiducial from the root- end. There are four strain gauges in line at the bottom showing as light spots with the fiducial half way between the center two. The large white hot oval is the location of the root of insert \#12 while the smaller hot spot to its right is the root of insert \#11.
Figure 3 shows the digital infrared camera pixel profile across the line on figure 2 . The heat generated by the flexing around the roots of inserts $\# 11, \# 12$ and $\# 13$ is evident. This temperature profile is of the equilibrium temperature on the surface of the blade with about one-inch of wood laminates between the surface and the inserts.

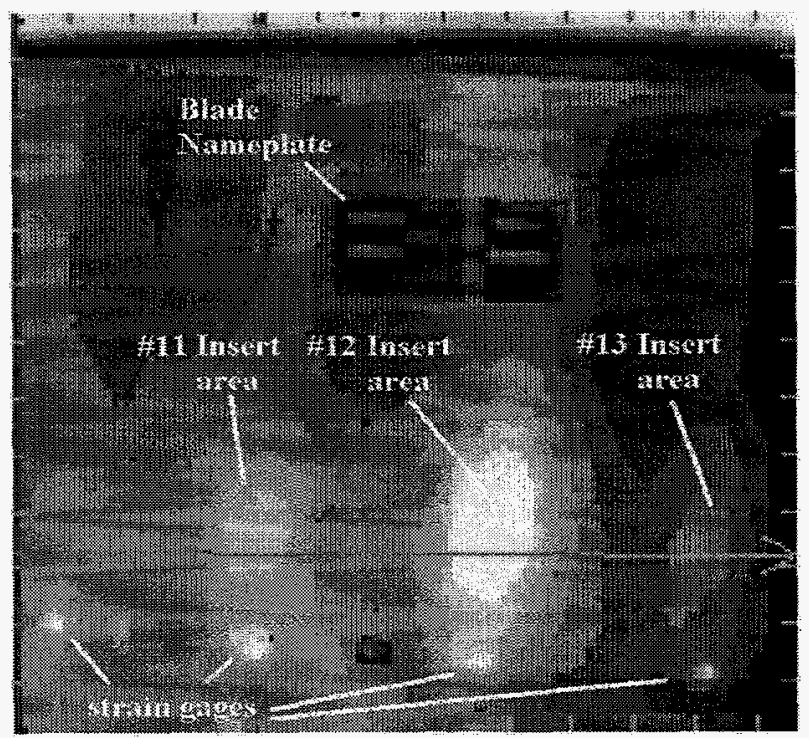

Figure 2. Steady-state (DC) thermal image of blade root area. The root-end is along the top. White areas are at higher temperatures than the dark areas.

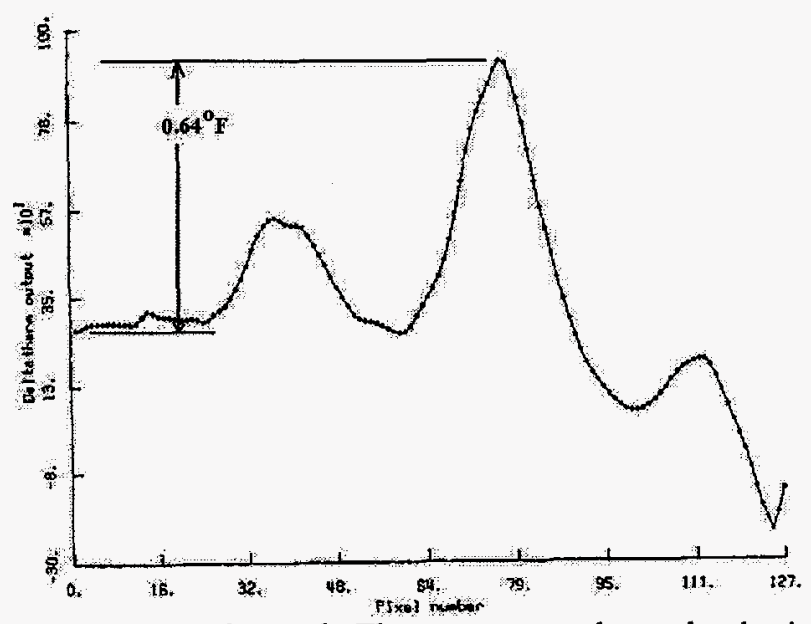

Figure 3. The DeltaTherm output along the horizontal line shown in the image in Figure 2.

After the infrared camera system was back in the laboratory, it was found that it is possible to calibrate the system so that one can express the steady-state (DC) thermal images in terms of actual temperatures. The thermometry used in the calibration is not as accurate as one might wish so no temperatures are given in figure 3. 
However, the temperature spread is more accurate than the actual temperature. The spread in figure 3 , running from the value at the left edge in figure 2 (pixel 0 ) to the value at the peak near pixel 77 , is $0.64^{\circ} \mathrm{F}$.

Figure 4 is a stress (AC) image of approximately the same area seen in figure 2. The shades of gray represent variations in the sum of the principal stresses on the blade surface. The multi-shaded line along the top is the edge of the blade root. The stress intensity is low at the end of the root where the studs are carrying most of the load and high at the ends of the studs where the load is transferred into the wooden blade. An increase in stress at inserts \#12 and \#13 are easily seen. Again, these are surface stresses with at least an inch of wood laminates between the surface and the highly stressed regions around the insert ends.

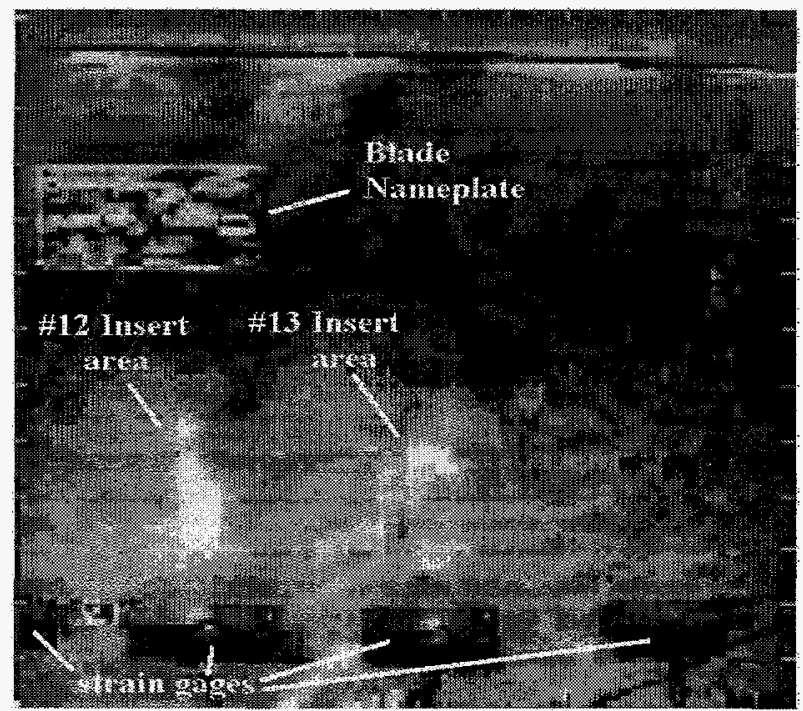

Figure 4. A stress (AC) image of the blade root area. The root-end is along the top. Lighter areas are at higher stresses.

The last feature that appeared in the stress data is seen in the image shown in figure 5. The almost horizontal line, seen about two thirds of the way up in the image, corresponds in position with a scarf joint between two pieces of laminate on the bottom of the blade. There was no sign of this horizontal line in the steady-state (DC) image. It is thought that this line is a small stress perturbation caused by the joint in the surface wood just under the gelcoat epoxy finish. Closer visual observation did not identify any surface imperfection that might cause a reflection at this location. However, the portion of the line extending left of the vertical reflection stripe in figure 5 does show some mottling. The best interpretation is that the line in the center is produced by a stress discontinuity at the joint, but some of the line at the left is probably due to a shallow dimple in the epoxy surface caused by the joint. [Figure 5 was obtained early in the project; we were still learning about how to deal with the reflections. Bright infrared reflections did not always have a significant visible reflection! As an example, the vertical stripes on the left are bright infrared reflections of warm hydraulic hoses (that are visibly black) lying on the concrete floor. The vertical reflection in the center is the 2 " $x 4$ " board of pine that is part of the floor-mount slide for the infrared camera.]

This blade ultimately failed in the root region around the area of insert $\# 12$ at 714,926 cycles.

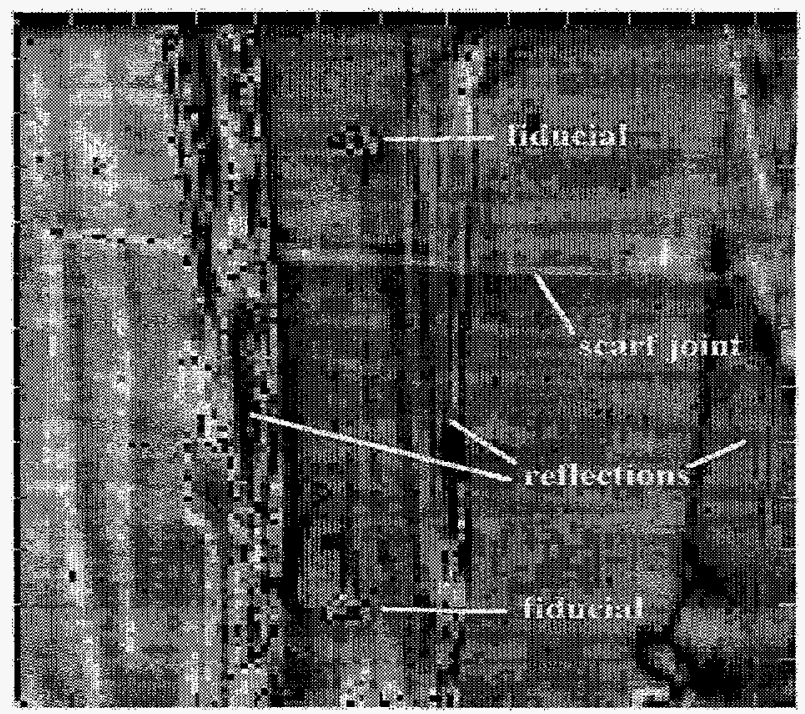

Figure 5. A stress (AC) image of the blade showing a scarf joint.

\section{Pultruded Blade Test}

For the pultruded blade fatigue test, the test specimen was a section of a FloWind "B-blade" wind turbine blade. The blade was made of pultruded fiberglass reinforced plastic, with a constant chord of 0.685 meters. Two internal spars, or shear-webs, ran down the span of the blade. In a previous test, the blade had been subjected to a large number of cycles at a resonant frequency of $23.75 \mathrm{~Hz}$. The accelerations in this test gave force levels up to $100 \mathrm{G}$ on portions of the blade. Spanwise cracks near the trailing edge developed at one end. The region containing the cracks was cut off and the remaining 4.25 meters was subjected to modal analysis ${ }^{4}$. As was done in the previous test, the 4.25 meter section of blade was suspended by two straps attached to an overhead a crane, as shown in Figure 6. The test setup had the chord plane vertical with the trailing edge up. The electromagnetic driver was horizontal and coupled to the blade by a stinger consisting of a thin plastic rod. This rod was attached both to the 
driver and to an aluminum pad glued to the rear surface of the blade. The pad was located near the right hand corner of the blade close to the trailing edge $(6.4 \mathrm{~cm}$ down and $2.5 \mathrm{~cm}$ in from the blade edge). (Figure 6 shows the driver attached at a different location than the one used in the experiment. The driver is not visible, but the tripod and legs supporting the driver is visible, right of center.)

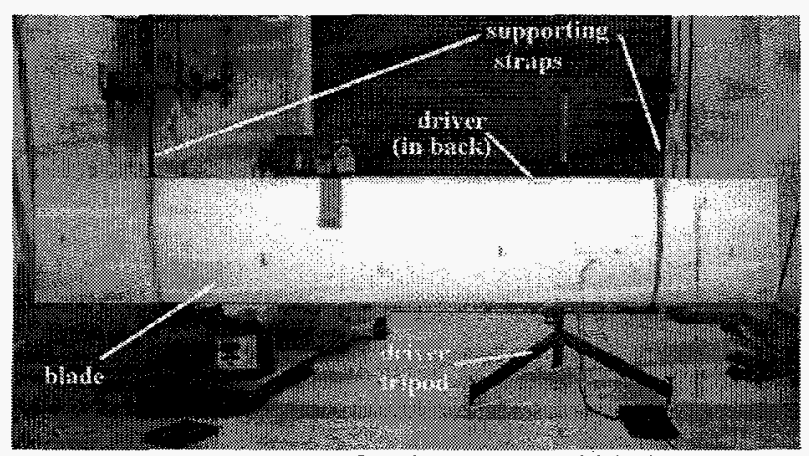

Figure 6. Test setup for the pultruded blade test.

A function generator was used to generate the sinusoidal drive waveform, and to set the frequency at an integral number of Hertz. The waveform was amplified and sent to the electromagnetic driver. The drive amplitude was arbitrary and set to a level where the blade was obviously resonating. The frequencies were initially taken from reference 4 for the desired modes, then adjusted to the nearest integral frequency that had largest amplitude of motion. Frequencies of 38.0 and 78.0 $\mathrm{Hz}$ were used.

The digital infrared camera takes a square image. The long narrow blade required a series of images to cover it entirely. The images were taken with a distance of 2.9 meters between the lens and the blade. Six images were taken for each different $\mathrm{AC}$ frequency and combined into a composite picture. A similar series of steady-state (DC) thermal images were also taken while the blade was being driven. The procedure used was to move the camera parallel to the blade a predetermined distance from the previous location. (The junction line between floor tiles was used as a guide to determine the camera position.) The alignment between adjoining images was not perfect, probably due to minor changes in the tilt angles of the camera and parallax.

In the stress ( $\mathrm{AC}$ ) measurements, the phase angle between the drive frequency and the best in-phase image was determined. For these tests, the phase angle that gave the maximum separation between the in-phase signal and one $90^{\circ}$ away was determined from the image of the far right section of the blade. This phase angle was arbitrarily used for all other images taken at that frequency. For the steady-state (DC) measurement, the blade was driven at $38 \mathrm{Hertz}$ for two hours to allow the blade to reach thermal equilibrium.

When an image is displayed, the computer automatically assigns 16 colors to different amplitudes over a rather arbitrary range. These assignments differ somewhat for each separate image. However, the operator is able to adjust this color range for each image. In order to try to join the images into a single panorama of the blade, the color assignments were set the same for each image in the set. The six separate images were then digitally joined into one image of the blade. The stress $(A C)$ images were all given the same color range although small displacements in the center of the range of each image were used to improve the match between images. The stress ( $A C$ ) images show only the relative differences between the maximum and minimum temperatures, referenced to the average blade temperature, which are in synchronization with the drive frequency. This almost completely removes the differences in DC temperatures between images and allows a relatively good composite picture.

On the other hand, the steady-state (DC) images show the temperatures with a reference image, taken at a slightly different time, subtracted from the original image. To achieve high-resolution temperature, an outof-focus image of a black aluminum block, located near and at approximately the same ambient temperature as the blade, was used as the temperature reference. This removes both differences in the gains of the individual detectors and the large steady-state (DC) thermal signal produced by the absolute temperature of the sample. Thus temperature differences of a few hundredths of a degree can easily be seen. However, small drifts in the absolute temperature or in the temperature gradient across the image in either image can produce different temperature scales and fictitious temperature gradients in the corrected image. This makes the steady-state (DC) images much more difficult to combine into one image of the blade than the stress (AC) images. Another problem with the steady-state (DC) images is that they often have the appearance of a coarsely woven carpet. [The reason for this was not fully understood, but the problem worsened, months later, to the point where the camera required repair.]

Figures 7A and 7B show steady-state (DC) and stress (AC) images, respectively, for the fundamental bending mode at $38 \mathrm{~Hz}$. The steady-state (DC) thermal images do not fit together perfectly, but do show a rather smooth temperature distribution. \{The carpet weave appearance is readily seen in the steady-state (DC) thermal images.\} The stress (AC) image shows a much better coherence between the separate images than does the steady-state (DC) image. 


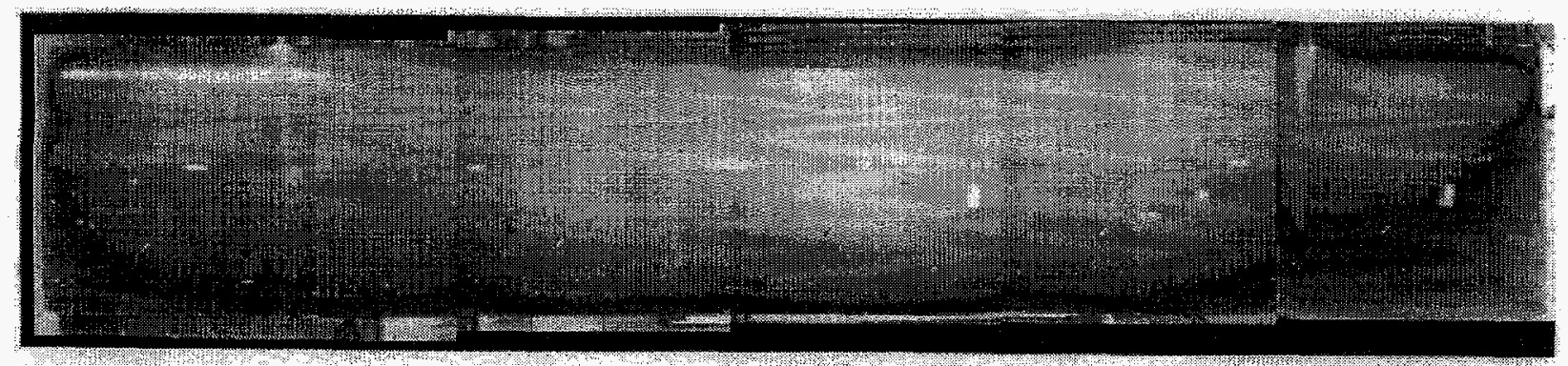

Figure 7A. Steady-state (DC) thermal image of pultruded blade excited by the 38 Hertz mode.

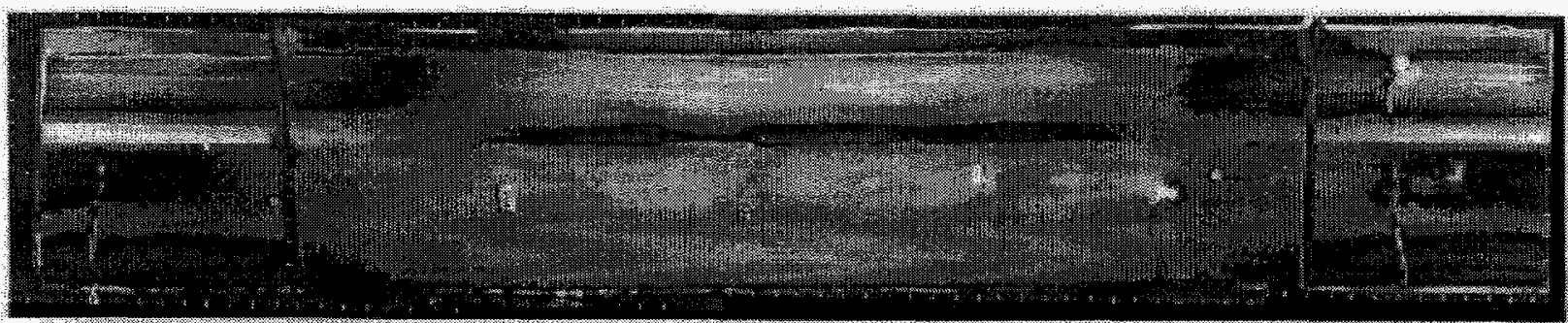

Figure 7B. Stress (AC) image of pultruded blade at the 38 Hertz mode.

Figure 8 shows a finite element calculation of the $38 \mathrm{~Hz}$ mode for the blade, showing the relative amplitudes of the sum of the principal stresses on the surface. The correspondence between the patterns seen on the blade, in the composite stress (AC) picture, and the finite-element calculation, is remarkably good.

The center of the blade shows a resonance in the skin between the rear spar or webbing and the trailing edge. The steady-state (DC) thermal image shows a relatively smooth temperature distribution with no hint of the skin resonance. The bright horizontal line near the left trailing edge in this image is a crack and will be discussed later.

Figure 9A shows the steady-state (DC) thermal images, driven at $78 \mathrm{Hertz}$, at just the ends of the blade
( 2 of the normal 6 images). Figure 9B shows the stress (AC) patterns at a resonance frequency of $78 \mathrm{Hertz}$. The $78 \mathrm{Hertz}$ resonance is supposed to be the second harmonic of the 38 Hertz resonance. However, the stress (AC) picture shows the double peak lying between the suspending straps instead of covering the whole length of the blade. Here, the resonance in the skin between the rear spar and the trailing edge becomes very prominent. The steady-state (DC) thermal images of the ends show a pronounced hot spot on the left end and a very thin hot line on the right. Dye-penetrant analysis showed a major crack structure on the left end and a minor crack on the right. [The right end was the end that was cut off after a previous test. The left-side crack was either not noticed after the previous test or had not formed yet.]

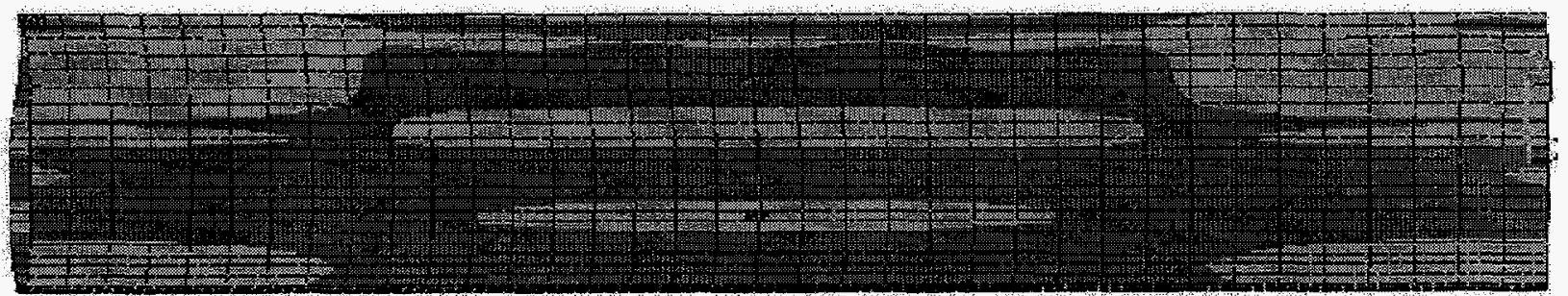

Figure 8. Finite-element calculation of the relative modal stress patterns at the 38 Hertz resonance. 


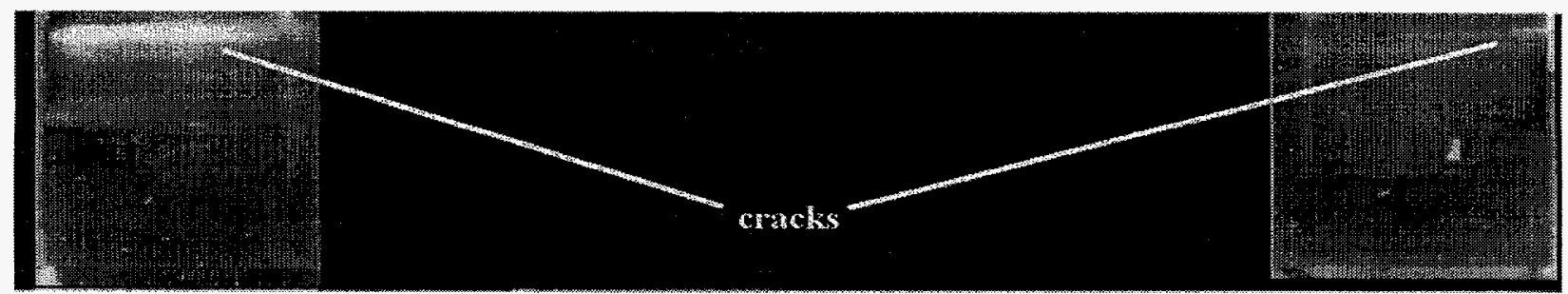

Figure 9A. Steady-state (DC) thermal image of pultruded blade excited by the 78 Hertz mode.

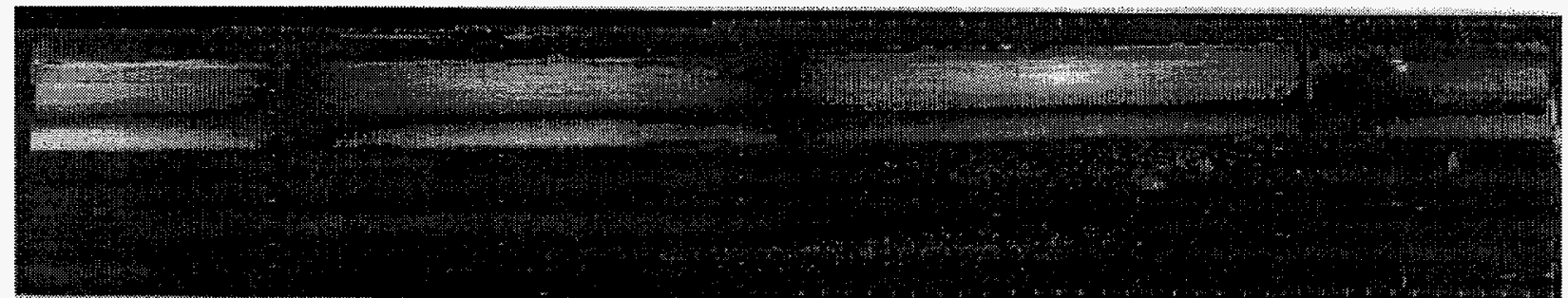

Figure 9B. Stress (AC) image of pultruded blade at the 78 Hertz mode.

Figure 10 shows close-up steady-state (DC) and stress (AC) thermal images of the left-end trailing edge of the blade. The steady-state (DC) image shows intense local heating while the stress (AC) image shows a stress pattern of a complex crack. (The infrared camera was about 1.1 meters away from the blade. Each camera pixel covered about a 2 by $2 \mathrm{~mm}$ square. The distance between the crack and the blade trailing edge was about $76 \mathrm{~mm}$.) The geometry is almost identical to the crack pattern obtained in the dye-penetrant analysis in this same area. The small very thin hot line, near the trailing edge, on the right end in the steady-state (DC) image, in figure 9A, was also shown to correspond to a small crack in the dyepenetrant analysis.
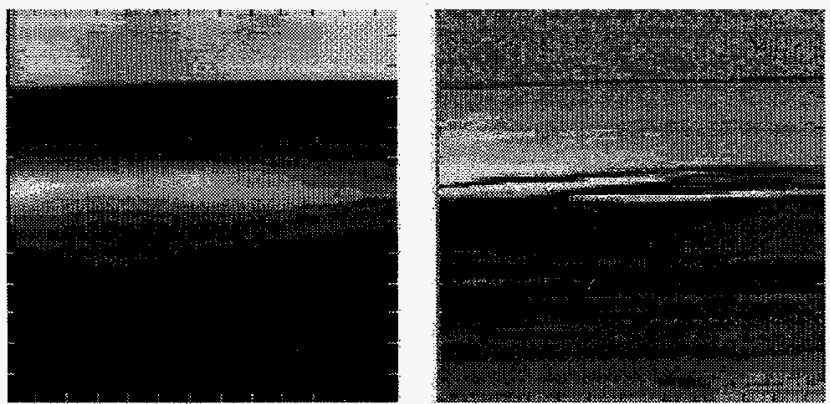

Figure 10. Close-up steady-state (DC) thermal and stress (AC) images, at 78 Hertz resonance, of the crack in the left trailing-edge end of the pultruded blade.

\section{Summary}

The DeltaTherm $1000^{\mathrm{TM}}$ digital infrared camera was able to predict the region where failure ultimately occurred in the root area of the AWT blade during a fatigue test. These measurements were taken at $14 \%$ of the fatigue life of the blade. The steady-state (DC) thermal image showed localized heating between the wood-epoxy-composite blade material and the metal insert. This heating was detected through approximately $3 \mathrm{~cm}$ of wood. There was also a small increase in the surface stress state seen on the surface above the inserts. Around the middle of the blade, a small perturbation in the stress level, above a scarf joint, was also seen.

The test of the pultruded blade section demonstrated that modal vibration patterns of a blade could be determined experimentally with a digital infrared camera by driving the blade into a resonance and taking a set of synchronized stress (AC) images of the whole blade. The modal pattern images seen were in good agreement with a finite-element calculation; the digital infrared camera images did appear to contain somewhat more detail than did the calculation. Finally, surface cracks could be located from the intense local heating produced by rubbing. The detailed surface geometry of the crack could then be determined by close-up stress (AC) images.

Dealing with infrared reflections complicated the test setup; the reflections were traced down and reduced or eliminated by shielding or simply moving the infrared source. 


\section{Conclusions}

This project has demonstrated that using a digital infrared thermography camera as a non-contact wide-area technique to detect steady-state thermal and thermoelastic stress effects can yield a significant amount of information that otherwise would be difficult to obtain. The camera obtained information on large and small areas of the blade. Blade material under the intense activity areas eventually failed (primary failure sites); areas that were not as active (possible secondary failure sites) could be significant in understanding the long-term life characteristics of the component.

\section{Acknowledgment}

The authors would like to thank Mike Jenks and the rest of the staff at the structural blade testing facility located at the NREL/NWTC facility for their assistance, support and interest during the monitoring of the AWT blade fatigue test. At Sandia National Laboratories (SNL), Dave Kelton, of the Experimental Structural Dynamics Department, gave enthusiastic technical assistance in setting-up and running the pultruded blade resonant fatigue test. We would like to also acknowledge Daniel Laird, of the SNL Wind Energy Technology Department, for the finite-element calculations using his NuMAD blade analysis tool; and to Tom Carne, of the SNL Experimental Structural Dynamics Department, for his advice and encouragement.

\section{References}

1) "Thermoelastic Stress Analysis," ed. N. Harwood and W.M. Cummings, Philadelphia, A. Hilger, c1991.

2) The SPATE system is manufactured by Ometron Inc., 502 Shaw Rd. B101, Dulles, VA. 20166-9435

3) Stress Photonics Inc., 3002 Progress Rd., Madison, WI 53716

4) "Estimation of Uncertain Parameters using Modal Test Data," P.S. Veers, D.L. Laird, T.G. Carne, and M.J. Sagartz AIAA-98-0049 